\title{
152K-computer-node parallel scalable implicit solver for dynamic nonlinear earthquake simulation
}

\author{
Tsuyoshi Ichimura \\ ichimura@eri.u-tokyo.ac.jp \\ Earthquake Research Institute \& \\ Department of Civil Engineering, The \\ University of Tokyo, \\ Center for Advanced Intelligence \\ Project \& Center for Computational \\ Science, RIKEN \\ Japan \\ Ryota Kusakabe \\ Yuma Kikuchi \\ ryota-k@eri.u-tokyo.ac.jp \\ kikuchi-y@eri.u-tokyo.ac.jp \\ Earthquake Research Institute \& \\ Department of Civil Engineering, The \\ University of Tokyo \\ Japan \\ Lalith Maddegedara \\ lalith@eri.u-tokyo.ac.jp \\ Earthquake Research Institute \& \\ Department of Civil Engineering, The \\ University of Tokyo \\ Japan
}

\author{
Kohei Fujita \\ fujita@eri.u-tokyo.ac.jp \\ Earthquake Research Institute \& \\ Department of Civil Engineering, The \\ University of Tokyo, \\ Center for Computational Science, \\ RIKEN \\ Japan
}

\author{
Takane Hori \\ horit@jamstec.go.jp \\ Research Institute for Marine \\ Geodynamics, Japan Agency for \\ Marine-Earth Science and Technology \\ Japan
}

\author{
Kentaro Koyama \\ kkoyama@fujitsu.com \\ Fujitsu Ltd. \\ Japan
}

oriyuki Ohi

Tatsuo Nishiki

Hikaru Inoue

n.ohi@fujitsu.com

nishiki.tatsuo@fujitsu.com

inoue-hikaru@fujitsu.com

Fujitsu Ltd.

Japan

Naonori Ueda

naonori.ueda@riken.jp

Center for Advanced Intelligence

Project, RIKEN

Japan

\author{
Muneo Hori \\ horimune@jamstec.go.jp \\ Research Institute for \\ Value-Added-Information Generation, \\ Japan Agency for Marine-Earth \\ Science and Technology \\ Japan
}

\begin{abstract}
We have used data learning and low-precision computation to develop an implicit solver that demonstrates high performance up to 152,352 computer nodes $(609,408$ MPI processes $\times 12$ OpenMP

Permission to make digital or hard copies of all or part of this work for personal or classroom use is granted without fee provided that copies are not made or distributed for profit or commercial advantage and that copies bear this notice and the full citation on the first page. Copyrights for components of this work owned by others than ACM must be honored. Abstracting with credit is permitted. To copy otherwise, or republish, to post on servers or to redistribute to lists, requires prior specific permission and/or a fee. Request permissions from permissions@acm.org.

HPC Asia2022, January 12-14, 2022, Virtual Event, Japan

(c) 2022 Association for Computing Machinery.

ACM ISBN 978-1-4503-8498-8/22/01 ..\$15.00

https://doi.org/10.1145/3492805.3492814
\end{abstract}

threads $=7,312,896$ parallel computation) and conducted an unprecedented ultra-large-scale analysis of ultra-high-fidelity faultstructure systems using nonlinear dynamic finite element analysis on three-dimensional low-order unstructured elements. The developed solver achieved 25.45-fold speedup from the state of the art solver on Fugaku and attained weak scaling efficiency of 93.7\% from 9.391 billion DOF@578 computer nodes to 1.201 trillion DOF@73,984 computer nodes on performance measurement problems. Moreover, a realistic 324 billion DOF application example, which is difficult to obtain performance for, was computed in high performance. Since the developed solver is based on a highly generalizable algorithm, it is expected to contribute not only to earthquake simulation on Fugaku but also to the enhancement of similar applications in other fields and on other supercomputers. 


\section{CCS CONCEPTS}

- Computing methodologies $\rightarrow$ Massively parallel algorithms; Massively parallel and high-performance simulations; $\bullet$ Applied computing $\rightarrow$ Earth and atmospheric sciences.

\section{KEYWORDS}

Massively parallel computation, finite-element method, fault-structure earthquake simulation

\section{ACM Reference Format:}

Tsuyoshi Ichimura, Kohei Fujita, Kentaro Koyama, Ryota Kusakabe, Yuma Kikuchi, Takane Hori, Muneo Hori, Lalith Maddegedara, Noriyuki Ohi, Tatsuo Nishiki, Hikaru Inoue, Kazuo Minami, Seiya Nishizawa, Miwako Tsuji, and Naonori Ueda. 2022. 152K-computer-node parallel scalable implicit solver for dynamic nonlinear earthquake simulation. In International Conference on High Performance Computing in Asia-Pacific Region (HPC Asia2022), January 12-14, 2022, Virtual Event, Japan. ACM, New York, NY, USA, 12 pages. https://doi.org/10.1145/3492805.3492814

\section{INTRODUCTION}

As the threat of disasters to cities and society increases, the rapid transformation of cities and society in terms of digital transformation is further accelerating toward the post/with corona era. In a situation where urban environments have changed significantly from previous disasters, it is difficult to expect an adequate damage reduction simply by relying on damage experience. Conversely, because of the digital transformation of cities and society, more detailed urban and social data are now available than ever before. By combining past damage experience and simulations using highly detailed urban/social data, we can expect the realization of reliable damage mitigation measures that are significantly improved from those of the past. However, this analysis is of large scale and has not been enabled yet; hence, the contribution from the supercomputing field is indispensable for this realization.

In this study, we focus on earthquakes, which are natural disasters that destroy cities and society and result in the loss of lives and property. There are numerous examples of damaging earthquakes worldwide, and major earthquakes are feared to occur soon; for example, an earthquake of the same magnitude as the 1906 San Francisco earthquake is expected on the west coast of the United States and in Tokyo, and an earthquake of the same magnitude as in 2011 Northeastern Japan earthquake is expected in the Cascadia subduction zone of Northern California and Southwestern Japan, which is a huge risk to the current urban society. An earthquake is a dynamic nonlinear problem that comprises i) seismic waves generated by fault rupture process propagating deep underground; ii) waves amplified in the sedimentary layer near the earth surface; and iii) amplified seismic waves strongly shaking cities, society, and structures (see Fig. 1). Contributions from the supercomputing field has been anticipated in the past to such a socially significant problem, and various results have been produced (e.g., [1]). For example, [2-8] focused on solving i); $[9,10]$ on solving ii); and [11] on solving ii) and iii) were presented at the Supercomputing Conferences, and they are expected to contribute greatly to the improvement of analysis for earthquake science and mitigation.

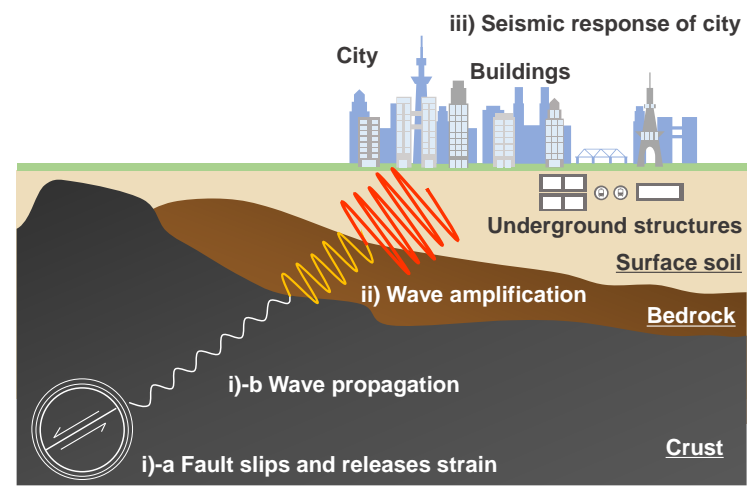

Figure 1: Earthquake processes. i) Wave propagation in crust due to fault rupture; ii) nonlinear wave amplification in sedimentary layers near the surface of the earth; and iii) strong shaking of cities, society, and structures. Fully coupled analysis of i)-iii) is desired but has been considered difficult because of the enormous analysis cost.

By contrast, there is a completely untouched problem. Originally, in this seismic simulation, it is necessary to solve ultrahigh-fidelity fault-structure systems (the above i)-iii)) all at once, which is equivalent to solving a nonlinear dynamic problem of size $10^{5-6} \times 10^{5-6} \times 10^{4-5} \mathrm{~m}$ with the minimum resolution of $10^{-1} \mathrm{~m}$ corresponding to the resolution of structures. As a method that satisfies the stress-free boundary conditions along the surface and can evaluate the stress response for modeling complicated and fine geometry of structures, we consider analysis by a three-dimensional (3D) dynamic nonlinear finite element method using unstructured second-order tetrahedral elements with implicit time integration. Note that if a super-complex geometry is accurately modeled with solid elements, small elements will be generated locally. The computation cost becomes huge when using explicit time integration because small time steps must be used to ensure stability; thus, it is necessary to use stable implicit methods. Consequently, this problem is equivalent to solving a nonlinear dynamic 3D finite element analysis problem of $10^{11-13}$ degrees of freedom (DOF) and $10^{3-4}$ time steps using implicit time integration (i.e., Newmark- $\beta$ method [12] with $\beta=1 / 4$ and $\delta=1 / 2$ ) with an iterative solver with double precision variables.

$$
\begin{array}{r}
\left(\frac{4}{d t^{2}} \mathbf{M}+\frac{2}{d t} \mathbf{C}^{n}+\mathbf{K}^{n}\right) \delta \mathbf{u}^{n}= \\
\mathbf{F}^{n}-\mathbf{Q}^{n-1}+\mathbf{C}^{n} \mathbf{v}^{n-1}+\mathbf{M}\left(\mathbf{a}^{n-1}+\frac{4}{d t} \mathbf{v}^{n-1}\right),
\end{array}
$$

where, $\delta \mathbf{u}, \mathbf{u}, \mathbf{v}, \mathbf{a}$, and $\mathbf{F}$ represent the incremental displacement, displacement, velocity, acceleration, and external force vector, respectively. M, C, and $\mathrm{K}$ represent the consistent mass, damping, and stiffness matrix, respectively. $d t$ and $n$ denote time step increment and time step number. In this study, the Rayleigh damping matrix is used for $\mathrm{C}$, where the element damping matrix $\mathrm{C}_{e}^{n}$ is given as Rayleigh damping using the consistent element mass matrix $\mathbf{M}_{e}$ and the element stiffness matrix $\mathbf{K}_{e}^{n}$. Using $\delta \mathbf{u}^{n}$ obtained from solving Eq. (1) and constructing the stiffness matrix considering nonlinearity for 
each time step, the nonlinear time history problem is solved by updating the variables as $\mathbf{Q}^{n} \Leftarrow \mathrm{Q}^{n-1}+\mathrm{K}^{n} \delta \mathbf{u}^{n}, \mathbf{u}^{n} \Leftarrow \mathbf{u}^{n-1}+\delta \mathbf{u}^{n}$, $\mathbf{v}^{n}=-\mathbf{v}^{n-1}+\frac{2}{d t} \delta \mathbf{u}^{n}$, and $\mathbf{a}^{n} \Leftarrow-\mathbf{a}^{n-1}-\frac{4}{d t} \mathbf{v}^{n-1}+\frac{4}{d t^{2}} \delta \mathbf{u}^{n}$. Solving the $10^{11-13}$ DOF problem of Eq. (1) with poor convergence for $10^{3-4}$ time steps is positioned as an ultimate earthquake simulation that is far more challenging than the state-of-the-art problems in i)-iii). For a long time, this simulation has been tackled in various ways, but because the amount of computation is huge, several attempts have been made to solve it with coarser models or by dividing it into i), ii), and iii) (e.g., [13-15]). For example, one of the SC15 Gordon Bell Prize Finalists [10] used the entire K computer system at full scale using a method based on [15] by multi-scale analysis based on singular perturbation, but they only reached the point where i)-iii) are divided and solved. i)-iii) are inextricably linked, and it is difficult to separate them in principle. The effect and outcome of breaking this link are unknown as it is a dynamic nonlinear problem. A fully coupled analysis of i)-iii) is desired, but as mentioned above, it is an unattainable ultimate earthquake simulation with an enormous amount of calculation, and there is no state-of-the-art solution. In this study, we aim to realize this ultimate earthquake simulation and open the frontier in the field of earthquake simulation.

Since the reliability of analysis results should be guaranteed to mitigate earthquakes in cities and society, considering various stakeholders, we develop a solver for 3D dynamic nonlinear finite element analysis via implicit time integration using a large number of unstructured second-order tetrahedral elements, as this method has been under quality assurance (e.g., V\&V[16]) in several fields such as manufacturing industry. Thus, this developed solver is expected to have broad ripple effects in many fields. For use in such fields, the development of an algorithm that is architecture independent and generalizable is essential in the sense that other scientists can learn and benefit from the innovations. Thus, we developed a solver that attains high performance of up to $152 \mathrm{~K}$-computer-nodes $(609,408$ MPI processes $\times 12$ OpenMP threads $=7,312,896$ parallel computation) by efficiently estimating higher-order modes while using the sparseness in the local region of the solution and the multiscale property of the solution space using general algorithms as much as possible. At the same time, for some special core kernels that are computationally rich but involve data recurrence and are often bottlenecks in other applications as well, we developed highly efficient implementations on various architectures that could be used as a reference for other applications. Additionally, various methods aimed at improving analysis efficiency by extracting knowledge from large-scale data generated during large-scale analysis have been proposed, but it is challenging to improve analysis efficiency while satisfying massively parallel efficiency. Thus, in this study, we have developed a data learning method that improves time to solution while achieving massively parallel efficiency and incorporated it into the developed solver (this data learning method can be easily introduced into other time evolution problems as well). The details of the above and the effectiveness of the proposed method are shown below using performance measurements on various architectures.

In this study, we successfully performed unprecedented ultimate earthquake simulation of a dynamic nonlinear fault-structure system with a domain size of $256000 \times 205000 \times 100000 \mathrm{~m}$ and a minimum resolution of $0.125 \mathrm{~m}$ using the proposed solver AEGIS (Advanced Earthquake General Integrated Simulator) on Fugaku (Fig. 2$)^{1}$.

For the performance measurement problem on Fugaku, AEGIS achieved a 25.45 -fold speedup compared with the state-of-the-art solver $P C G E^{K}$ (details given in ${ }^{2,3}$, with the compared solvers summarized in Table 1). Furthermore, $93.7 \%$ weak scaling efficiency was achieved from 9.391 billion DOF with 578 computer nodes to 1.201 trillion DOF with 73,984 computer nodes on the performance measurement problem. Although high efficiency was achieved with a realistic 324 billion DOF application example problem using 152,352 computer nodes, since full system jobs on Fugaku is planned to be limited to a few days per year, it is more important to solve application problems in the normal operation period (up to 55,296 nodes for $24 \mathrm{~h}$ ) in order to analyze various seismic scenarios. Thus, we also analyzed the application example (9550 time steps, $d t=$ $0.005 \mathrm{~s}$ ) using 52,992 Fugaku compute nodes (211,968 MPI processes $\times 12$ OpenMP threads $=2,543,616$ parallel computation), resulting in fast time to solution of $4 \mathrm{~h} 36 \mathrm{~min}$. If the state-of-the-art solver $P C G E^{K}$ is used to solve this application problem on Fugaku, it is expected to take $109.8 \mathrm{~h}$; thus, even with using the state-of-the-art solver, the analysis was difficult to conduct. We can see from the performance on the actual application problem that AEGIS is highly effective.

We show the detailed performance of AEGIS, the state-of-theart solver $P C G E$, and related solvers described above using an application example and performance measurement problems that resemble the application example in Section 3.

\section{METHOD}

We developed Algorithm 1 that is architecture independent and generalizable for fast massively parallel solving of Eq. (1) involved in 3D finite element analysis used in a wide range of fields. The above is achieved by efficiently predicting and reducing errors in higher-order modes, which can worsen convergence, and by enabling massive parallel computing while suppressing the influence of low-precision operations. Specifically, we extended the conjugate gradient method, which is a general implicit solver based on general operations that are architecture independent in order to incorporate a high-performance initial solution predictor that takes advantage of the sparsity of the solution in the local domain and a preconditioner that takes advantage of the multiscale nature of the solution

\footnotetext{
${ }^{1}$ Generation of this ultra-large-scale/ultra-high-fidelity finite element model was also a major challenge, but was accomplished by combining $[17,18]$.

${ }^{2}$ Initially, we anticipated that SC14 Gordon Bell Prize finalist solver GAMERA ${ }^{K}$ [9] developed for analysis on the $\mathrm{K}$ computer using low-precision computation can be used to solve this problem and planned to use its tuned version GAMERA ${ }^{F u g a k u}[20]$ and its accelerated version IRIS ${ }^{F u g a k u}$ [21] for solving this problem on Fugaku. However, since the problem size, as well as the ratio between the smallest and largest mesh sizes, was excessively large, the analysis became unstable because of the low-precision computation in the iterative solver when using the as-is method. Through various investigations, we found that $P C G E^{K}$, which is a simplified version of $G A M E R A^{K}$ that was developed on the K computer, can be regarded as the state-of-the-art solver that can stably compute such an unprecedented large-scale problem with high parallel efficiency. As the parallel efficiency of $P C G E^{K}$ is very high, we introduced a preconditioner and data learning scheme that can reduce the influence of the numerical error in low-precision computation and optimized it for Fugaku to develop a fast solver AEGIS suitable for massively parallel computation.

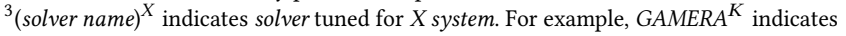
GAMERA tuned for the K computer.
} 


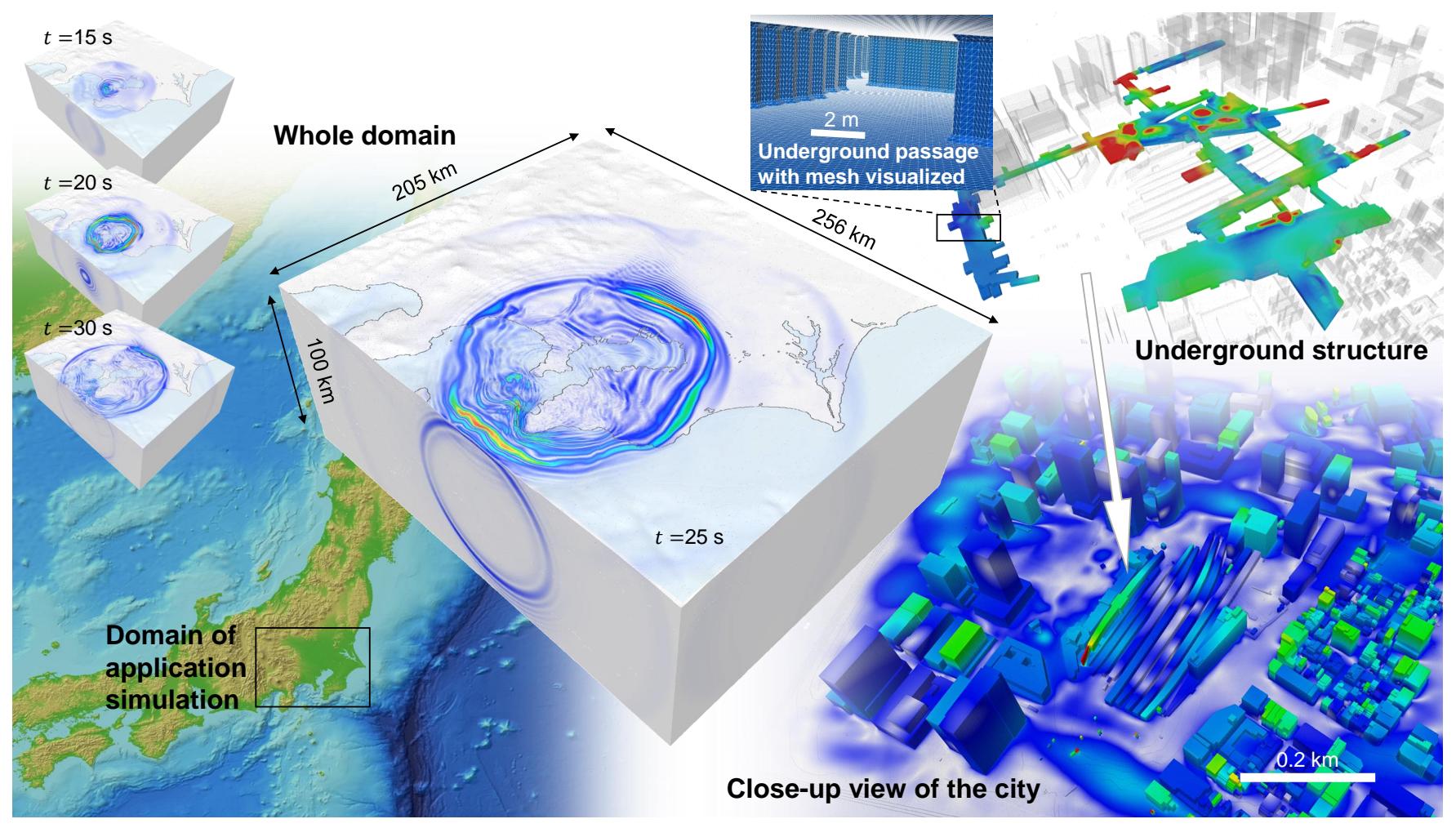

Figure 2: Three-dimensional low-order unstructured nonlinear finite element analysis of an inland earthquake on a faultstructure model comprising a highly integrated city near a terminal station with soil and crust of Kanto Plain in Japan. The application is an ultra-large-scale and ultra-high-fidelity problem: the domain size of the entire model is $256000 \times 205000 \times$ $100000 \mathrm{~m}$, discretization size varies from approximately 0.125 to $64 \mathrm{~m}$, the degree of freedom is $324,006,449,076$, and the number of second-order tetrahedral elements is 80,540,873,752. Enlarged view shows responses of the city with underground structures. This domain comprises soft ground layers on the heterogeneous crust, more than 1000 buildings, and an underground structure with more than 600 tenants. Complex geometries of structures are modeled in detail with a very fine mesh with a minimum discretization size of $0.125 \mathrm{~m}$. The bottom left map is from [19].

Table 1: Solvers compared in this study. CG method is abbreviation of Conjugate Gradient method. Note that the results obtained using the mixed-precision solvers are the same as double precision solver as low-precision computation is used only inside the solver preconditioners.

\begin{tabular}{|c|c|c|c|}
\hline & Solver algorithm & Computation precision & Kernel tuning \\
\hline$A E G I S$ & Developed data learning and multi-grid solver & Mixed precision & Kernel developed in this study for Fugaku \\
\hline$G A M E R A^{K}$ & CG method with multi-grid based preconditioner [9] & Mixed precision & Kernel developed in [9] for K computer \\
\hline GAMERA Fugaku & CG method with multi-grid based preconditioner [9] & Mixed precision & Kernel developed in this study for Fugaku \\
\hline$P C G E^{K}$ & CG method with $3 \times 3$ block Jacobi preconditioner & Double precision & Kernel developed in [9] for K computer \\
\hline PCGE $E^{\text {Fugaku }}$ & CG method with $3 \times 3$ block Jacobi preconditioner & Double precision & Kernel developed in this study for Fugaku \\
\hline
\end{tabular}

space and low-precision operations. Some special kernels with architecture dependency (i.e., element-by-element (EBE) [22] kernels for $\mathbf{K}, \mathbf{C}$, and $\mathbf{M}$ ) are required for implementing this algorithm, and acceleration with low-precision computation is essential; thus, we show that highly efficient implementations of these kernels are possible for each architecture and demonstrate that Algorithm 1 can be robustly executed with high efficiency on many systems. Here EBE is a type of kernel that is rich in computation, including data recurrence, which can be a bottleneck in general applications; thus, showing how to efficiently compute such a kernel is expected to be useful in other application development (as a concrete example and a reference for implementation, we plan to make the EBE kernel for A64FX CPU open source [23]). The details are as follows.

\subsection{Initial solution estimator}

There is a great demand for a predictor that estimates a better initial solution, reducing the number of iterations of the implicit solver. Here, the performance of the estimated solution against its analysis 
cost in a massively parallel environment becomes important. We construct an initial solution estimator with a small analysis cost suitable for massively parallel computation by building on the concept of dynamic mode decomposition (DMD) [24], which learns results up to $n$-1-th step and estimates the initial solution of the $n$ th step (used in line 3 of Algorithm 1). DMD estimates the operator of time evolution using a pseudo-inverse matrix of time series data of the solution; hence, the next solution is expected to be predicted from the current solution using this operator. However, estimating a large number of modes, including transit behavior, using DMD is costly, and stacking may be required when trend components are included, which further increases the analysis cost.

On the basis of the above, we develop a method suitable for massively parallel computing by estimating low-order modes, including the trend components, using low-cost analysis methods and estimating the remaining high-order modes in local space and time. Here, the analysis domain is divided into subdomains, and in each subdomain, the low-order mode solution $\delta \mathbf{u}_{a d a m}^{n}$, including the trend, is estimated using the Adams-Bashforth method using the velocity in the previous four steps as $\frac{d t}{24}\left(-9 \mathbf{v}^{n-4}+37 \mathbf{v}^{n-3}-59 \mathbf{v}^{n-2}+55 \mathbf{v}^{n-1}\right)$. Denoting the difference from the true solution $\delta \mathbf{u}^{n}$ as $\mathbf{x}^{n}=\delta \mathbf{u}^{n}-$ $\delta \mathbf{u}_{\text {adam }}^{n}$, we define matrix $\mathbf{X}^{n-1}=\left[\mathbf{x}^{n-1} \mathbf{x}^{n-2} \cdots \mathbf{x}^{n-s}\right]$ using the analysis results of the previous $s$ steps. Using matrix $\mathrm{X}$, the operator of time evolution $\mathrm{A}$ is defined as $\mathrm{X}^{n-1}=\mathrm{AX}^{n-2}$. The initial solution that includes both lower- and higher-order modes is computed as $\delta \mathbf{u}_{i n i}^{n}=\mathbf{A}\left(\delta \mathbf{u}^{n-1}-\delta \mathbf{u}_{\text {adam }}^{n-1}\right)+\delta \mathbf{u}_{\text {adam }}^{n}$. Unlike DMD, the modified Gram-Schmidt orthonormalization is used to compute $\mathrm{A}\left(\delta \mathbf{u}^{n-1}-\delta \mathbf{u}_{\text {adam }}^{n-1}\right)$. As the estimation is conducted in each subdomain, the number of modes (i.e., the number of learning data sets) required for predicting the behavior is reduced, and global calculation is avoided, enabling efficient massively parallel computation.

Where sufficient training data are available, the initial solution will be adequately estimated (e.g., in the performance measurement problem, the initial solution $\delta \mathbf{u}_{i n i}^{n}$ is estimated with a relative error of approximately $10^{-5}$ whereas $\delta \mathbf{u}_{\text {ad am }}^{n}$ is estimated with a relative error of approximately $10^{-3}$ ). As the estimation accuracy naturally deteriorates when training data are insufficient, the initial solution is automatically switched to $\delta \mathbf{u}_{a d a m}^{n}$ in such cases.

\subsection{Preconditioner}

Algorithm 1 is based on the inexact conjugate gradient method [25], which is a type of adaptive conjugate gradient method. Taking advantage of the adaptive conjugate gradient method, equation $\mathrm{A}^{n} \mathbf{r}=\mathbf{z}$ is solved with large tolerance in single precision using the conjugate gradient method as the preconditioner (Algorithm 1 line 8). Using $C G_{p r e}$ that solves $\mathrm{A}^{n} \mathrm{r}=\mathrm{z}, C G_{\text {pre }}^{\operatorname{lm} r}$ that solves a coarser problem that is equivalent to $\mathrm{A}^{n} \mathbf{r}=\mathbf{z}$ for estimating low-order modes, and $C G_{p r e}^{h m r}$ that solves a part of the domain where highorder modes are dominant for refining high-order modes, $\mathbf{A}^{n} \mathbf{r}=\mathbf{z}$ is solved with a modified w-cycle multigrid method that uses these solvers in the order of $C G_{\text {pre }}, C G_{\text {pre }}^{\operatorname{lm} r}, C G_{\text {pre }}, C G_{\text {pre }}^{l m r}$, and $C G_{p r e}^{h m r}$ Since it is a preconditioner, it can be solved approximately, and the low-order mode does not cause a problem even if it is handled roughly compared to the high-order mode; however, if high-order

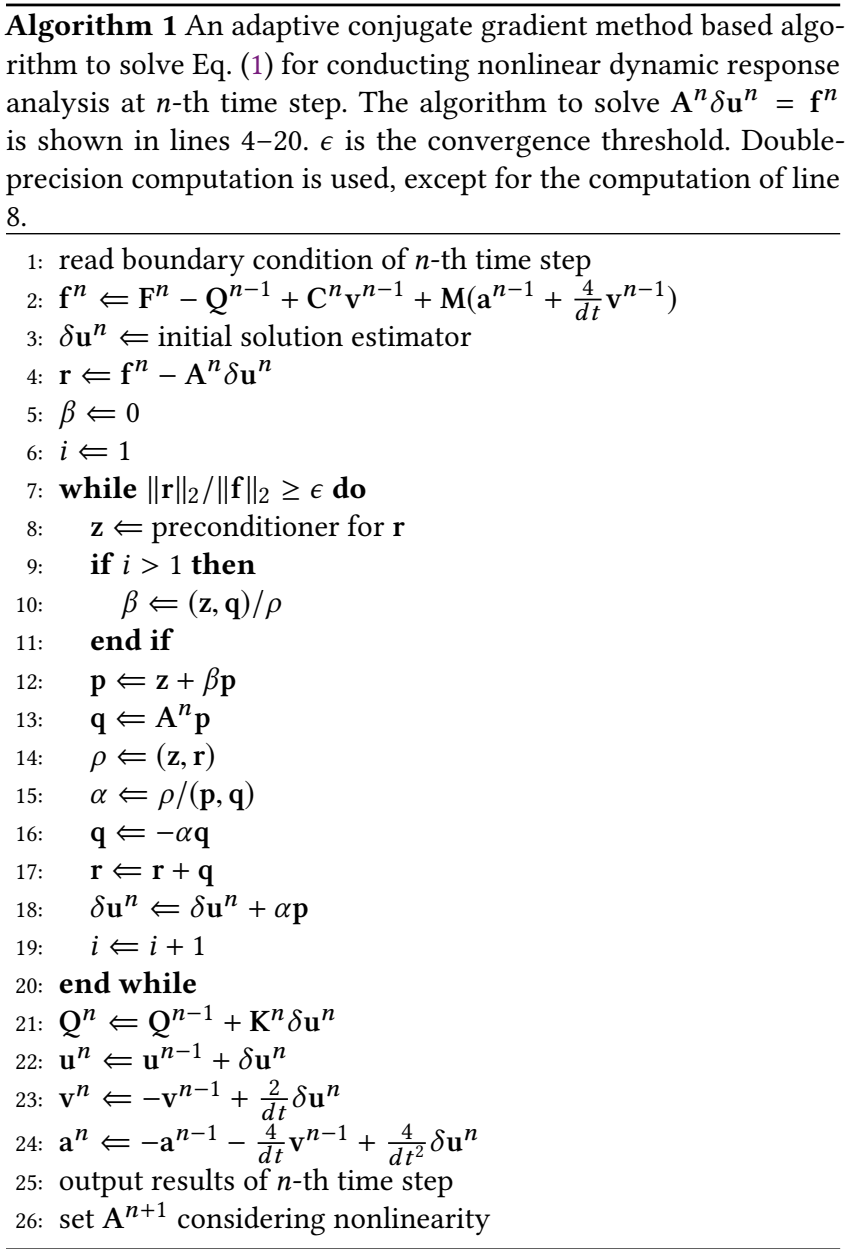

modes are not solved with a certain degree of accuracy, the convergence will deteriorate. Thus, designing the preconditioner considering the cost balance as described above is required for solving problems involving high-order modes with poor convergence as in the targeted problem. In this study, we solve $\mathbf{A}^{n} \mathbf{r}=\mathbf{z}$ with linear tetrahedral elements using the block Jacobi preconditioner in single precision for $C G_{p r e}^{\operatorname{lm} r}$. The coefficient matrix is stored in compressed row storage (CRS) format since high accuracy is not required for the computation of low-order modes and the DOF is smaller than that of $C G_{\text {pre }}$ ( $C G_{p r e}^{\operatorname{lmr}}$ is a standard CG solver using CRS). Since many iterations are involved in $C G_{\text {pre }}^{\operatorname{lm} r}$, it can be computed at high speed even when considering the CRS construction cost. Conversely, taking special care of the computation related to $\mathrm{A}^{n}$ in $C G_{\text {pre }}$ and $C G_{p r e}^{\mathrm{hmr}}$ are essential for suppressing the deterioration of accuracy in highorder modes while attaining fast computation speed. As the number of iterations of $C G_{p r e}$ and $C G_{p r e}^{h m r}$ is sufficiently small as high-order modes have been estimated accurately using the initial solution estimator, and since the DOF is large and thus the construction cost of CRS increases, computing $\mathrm{A}^{n}$ using fast EBE that is expected to suppress the deterioration of accuracy is required ( $C G_{p r e}$ and $C G_{\text {pre }}^{h m r}$ are also standard CG solvers, except for the terms related to $\mathrm{A}^{n}$ ). Although the EBE kernel is architecture-dependent and 
requires special consideration since it is a computationally rich kernel with data recurrence, there is no need to worry because there is a highly versatile implementation as shown next.

\subsection{Computationally rich kernels with data recurrence}

Contrary to the conventional matrix-vector product computation, which constructs the global matrix and reads it from memory during the matrix-vector product computation, the EBE method, which is a memory-free matrix-vector product computation method, constructs the element matrix $\mathrm{A}_{e}^{n}$ for each matrix-vector product computation, multiplies it by the element right-hand-side vector $\left(\mathbf{f}_{e}^{n} \Leftarrow \mathrm{A}_{e}^{n} \mathrm{~N}_{e}^{\mathrm{T}} \delta \mathbf{u}^{n}\right)$, and then, adds the result to the left-hand-side vector $\left(\mathbf{f}^{n} \Leftarrow \sum_{e} \mathrm{~N}_{e} \mathrm{f}_{e}^{n}\right)$. Here, $\mathrm{A}_{e}^{n}$ represents local element matrices computed from the nodal coordinates and material information, whereas $\mathrm{N}_{e}$ represents the matrices for the mapping of the elementwise nodal values to global nodal values. In the EBE method, since variables can be scaled locally for each element, it is easy to control the overflow and underflow of variables when using low-precision computation. Additionally, suppressing the accuracy degradation is possible by shifting values in advance for variables with close values that are subtracted from each other during element computation (e.g., suppressing the loss of digits is possible by subdividing the right-hand-side vector into the trend component and other modes for computation of the element stiffness matrix). Furthermore, in EBE, higher-precision arithmetic can be selectively applied to some operations that require particularly high precision (e.g., coordinate value computation), which allows for flexible adjustment of precision compared to matrix-vector products in the form of global matrix storage, where all operations must be performed in double precision to perform high-precision operations. Conversely, EBE has much more operations than the matrix-vector product in the global matrix storage format and requires a high efficiency computation, but it is not straightforward to be computed in parallel because it involves random additions with data recurrence. Thus, in this study, we design local kernel algorithms that suit each computer architecture for many-core and wide SIMD CPUs and GPUs that can perform fast atomic operations to achieve a general-purpose implementation (see [26, 27] for hardware specific algorithms for data recurrence).

In the following, the details are explained using the serial code of EBE in Fig. 3a. The index of $f$ in lines 18-20 (cny1-cny4) is irregular and recursive, resulting in data recurrence when adding values to the vector $f$, which inhibits parallelization in the ie loop. Although omitted in the figure, the computations in lines 14-16 are several hundred lines with variables closed within the loop; thus, if these lines cannot be computed in parallel, the performance of the kernel will deteriorate significantly. We first consider multithread parallelization of the ie loop in the CPU. The simplest way is to allocate temporary vectors for each thread $\left(f_{1}-f_{n t h r e a d}\right)$ and add results for each thread to the global vector $f$ after calculating all elements $\left(f=\sum_{\text {nthread }} f_{i}\right.$ ). Implementation is simple and easy, but in many-core environments with a large number of cores, the memory usage is high and the cost of aggregating the last threadlocal results is significant. Thus, following the example of [20], we developed a thread partitioning method to avoid data recurrence among multiple cores while maintaining cache efficiency (Fig. 3b). This method allows recurrence within the domain allocated to each thread, instead of decomposing the domain so that there is no overlap of nodes between all elements in each color as in the normal coloring shown in Fig. 3c, increasing the cache reuse rate. At the same time, we divided the element loop body into two loops, one for data reading and computation, and the other for data addition, so that SIMD operations can be applied in data reading and computation (Fig. 3d). By setting the size of the loop block NL to the same value as the SIMD width, the computation can be performed using the temporary buffer fe11-fe 34 on register or L1 cache (i.e., $\mathrm{NL}=16$ for single-precision operations on a 512-bit SIMD CPU, and $\mathrm{NL}=8$ for single-precision operations on a 256-bit SIMD CPU). In this way, many-core and wide SIMD arithmetic units can be used, and performance improvements can be expected in a wide range of CPU architectures. It is also possible to refine the method to suit the more detailed characteristics of the architecture; for example, speedup is achieved by further dividing the loops according to the number of registers, out-of-order resources, and operation/data access latency of the Fugaku's A64FX CPU and controlling the operation scheduling among the divided loops (this kernel for Fugaku will be available soon [23]).

In GPU computation, hiding data access and computation latency by launching a large number of threads simultaneously is essential to achieve high performance. However, the same threadpartitioning and loop-splitting approach as in the CPU version above would result in poor performance because of the large number of threads. Conversely, since recent NVIDIA GPUs are equipped with fast hardware-accelerated atomic addition instructions, we implemented a kernel that takes advantage of this function (Fig. 3e). Here, atomic addition is used only for the addition to $f$ that contains the data recurrence (lines 20-25). This atomic addition can be executed by the arithmetic unit adjacent to the distributed L2 cache, so it can be executed at high speed without the need to move data to the core. Additionally, further speedup is possible by aggregating the values of $f$ using the high-speed memory shared by multiple threads (e.g., shared memory) and then atomically adding aggregated values to the global memory, rather than directly atomically adding the values of all nodes to global memory. For GPUs that do not support hardware atomic additions, fast EBE calculation is possible by combining the above local value aggregations with the region decomposition method in Fig. 3b. By selecting one of the above EBE algorithms depending on the architecture, it is expected that the EBE kernel can be computed efficiently in various architectures.

\subsection{Massively parallel computation}

We incorporated ways to suppress performance degradation factors when solving ultra-large-scale problems as below.

Suppressing computational cost increase: Since the solution algorithm in this study is constructed using point-wise block Jacobi preconditioner-based CG methods, the computational cost for each iteration is proportional to the problem size. Thus, if the number of iterations of the CG method can be kept constant regardless of the problem size, good weak scaling can be expected for large-scale 

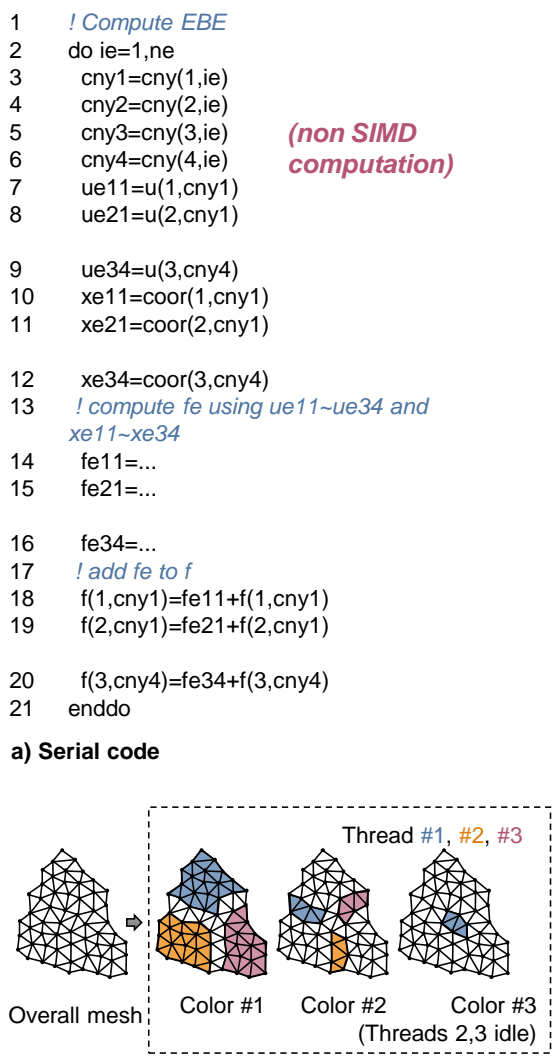

b) Thread partitioning for cache-efficient multithread parallelization

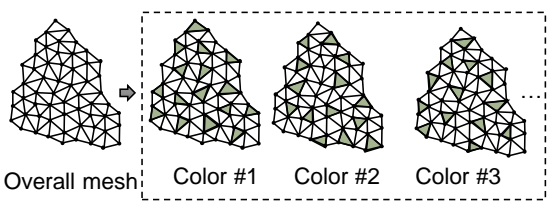

c) Coloring of mesh

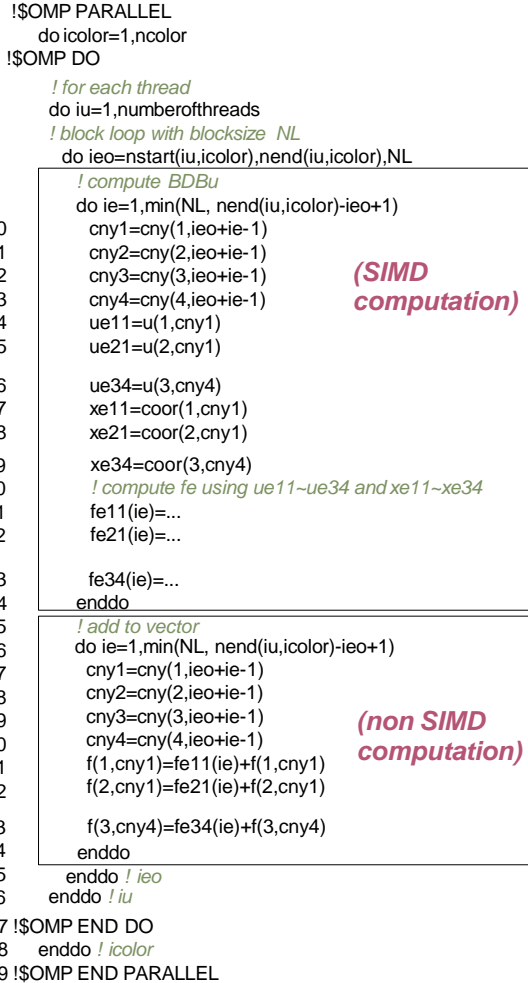

computation)

I \$acc parallel present(ne,cny,u,coor $f$ )

2 ! \$acc\& private(ie,cny1, cny2,cny3,cny4

3 ! \$acc\& ue11, ue 12, ..., ue 34 ,

4 ! \$acc\& xe11,xe21,.., xe34,fe $11, \mathrm{fe} 21, \ldots, \mathrm{fe} 34)$

5 do ie $=1$, ne

$6 \quad$ cny $1=\operatorname{cny}(1, \mathrm{ie})$

cny $1=\operatorname{cny}(1, \mathrm{ie})$
cny2 $=\operatorname{cny}(2, \mathrm{ie})$

cny3 $=$ cny $(3$, ie $)$

cny $4=\operatorname{cny}(4, \mathrm{ie})$

(SIMD $\quad \begin{array}{ll}10 & \mathrm{ue} 11=\mathrm{u}(1, \mathrm{cny} 1)\end{array}$

$\mathrm{ue} 21=\mathrm{u}(2$, cny 1$)$

\section{ue34=u(3,cny4)}

xe11 $=\operatorname{coor}(1$, cny 1$)$

xe21 $=\operatorname{coor}(2$, cny 1$)$

xe34=coor(3,cny4)

! compute fe using ue11 ue34 and xe11 xe34 fe $11=\ldots$

18 fe $21=\ldots$

19 fe34=...

20 !\$acc atomic

$21 \quad f(1$, cny 1$)=f e 11+f(1$, cny 1$)$

22 !\$acc atomic

$23 \mathrm{f}(2$, cny 1$)=f e 21+f(2$, cny 1$)$

24 ! \$acc atomic

$25 \quad f(3$, cny 4$)=f e 34+f(3$, cny 4$)$

26 enddo

27 ! \$acc end parallel

e) Tuned EBE kernel for GPU with hardware accelerated atomics

Figure 3: EBE kernel and thread partitioning/coloring for linear tetrahedral element. The code structure is the same for the targeted second-order tetrahedral element but with $10 \times 3=30$ components of ue and fe per element.

problems. If the accuracy of the solution is degraded because of lowprecision computation, the high-order modes cannot be captured, and the number of iterations is likely to increase. Thus, besides the measures to suppress the accuracy degradation of the matrixvector product in the EBE method, the scaling of the input vector was implemented to avoid loss of digits during global vector inner products. Additionally, the number of iterations of each CG solver in the w-cycle of preconditioning is limited to increase the number of synchronization of double-precision computation and prevent the solution from diverging.

Suppressing load imbalance: Although $C G_{\text {pre }}$ and $C G_{\text {pre }}^{\text {lmr cover }}$ the entire target domain, $C G_{p r e}^{h m r}$ selectively solves a part of $C G_{p r e}$ that has poor convergence. For this reason, it is necessary to partition the domain in such a way that good load balance is achieved in both $C G_{p r e}, C G_{p r e}^{l m r}$, and $C G_{p r e}^{l m r}$ during MPI computation. To solve this problem, the $C G_{\text {pre }}^{h m r}$ part is identified in advance as part of the model with poor convergence based on the mesh element connectivity and element material properties. Then, the $C G_{p r e}^{h m r}$ part and the remaining part are partitioned separately using graph partitioning software [28] to maintain good load balance while reducing the amount of communication.

Suppressing communication cost: In a massively parallel environment, besides global communication (i.e., MPI_Allreduce), the latency of neighboring communication tends to increase because of the increase in the number of communication hops, and it also tends to be more susceptible to noise from other jobs. Accurate estimation of the initial solution by learning from past solution data and efficient solution of the long-wavelength component by coarsegrid solvers reduce the total number of communication calls and suppress the effects of latency and noise. Furthermore, in Fugaku, 
some of the computer nodes process I/O of multiple nodes, so jobs are executed via node mapping that I/O nodes are used exclusively to prevent noise from other jobs.

\section{NUMERICAL EXPERIMENT}

We demonstrate the performance of AEGIS on finite-element models of a fault-structure systems by performance measurement on several computing environments as well as weak scaling and strong scaling measurements on Fugaku. We also confirm its performance in a real problem through measurements on an application example.

\subsection{Application used to measure performance}

We generated a finite element model from fault to urban structures mimicking the application example shown in Section 1 and measured the time required for nonlinear seismic wave computation. Here, we use a crustal and urban integrated model of $65500 \times 65500$ $\times 6000 \mathrm{~m}$, which was created based on digital data of the actual crust, ground, and structures around Tokyo (Fig. 4). The urban area is modeled with a minimum element size of $0.25 \mathrm{~m}$ to resolve the complex geometry of the structure and to ensure the convergence of the numerical solution even when the soft ground behaves nonlinear, whereas the crust is modeled with a minimum element size of $32 \mathrm{~m}$ to ensure the convergence of the numerical solution, reflecting the crustal structure. The amplitude of the seismic motion [29] observed at JMA Kobe during the 1995 Hyogo-ken Nanbu earthquake is reduced to $1 / 10$ and input to the bottom of the model with a time step increment of $d t=0.005 \mathrm{~s}$. As shown in Fig. 4, the target model has a wide range of properties and element sizes, making it a challenging problem to be solved efficiently using iterative methods because of its poor properties compared to conventional crust- or city-only seismic simulations. The above model is mirrored and connected in the $x$ and $y$ directions to generate weak scaling models with similar convergence characteristics (Table 2). We measure the number of iterations and execution time during the 401-600 time steps, which is when the seismic motion reaches the urban area and the convergence of the iterative solver started to deteriorate.

The performance of AEGIS and the state-of-the-art solver, PCGE, are compared in the performance measurement problem. $P C G E$ is equivalent to a solver that uses Adams-Bashforth for the initial solution estimation of line 3 in Algorithm 1 and a double-precision $3 \times 3$-block Jacobi preconditioner for the preconditioner of line 8. Since the matrix-vector product, which is the main operation, is performed in EBE, the method has excellent load balance and can robustly and rapidly solve a wide range of large-scale problems in a massively parallel environment. Each MPI partition is further partitioned using graph partitioning software, resulting in approximately 6,500 second-order tetrahedral nodes per subdomain for the initial solution predictor in AEGIS (data of previous $s=16$ time steps are used for the predictor). Since the time required to evaluate the nonlinear constitutive law (in this paper, a typical material nonlinearity model $[30,31]$ is considered) varies with the target problem, we compare the performance of the solver part, including the initial solution estimator, which can be compared for other problems.
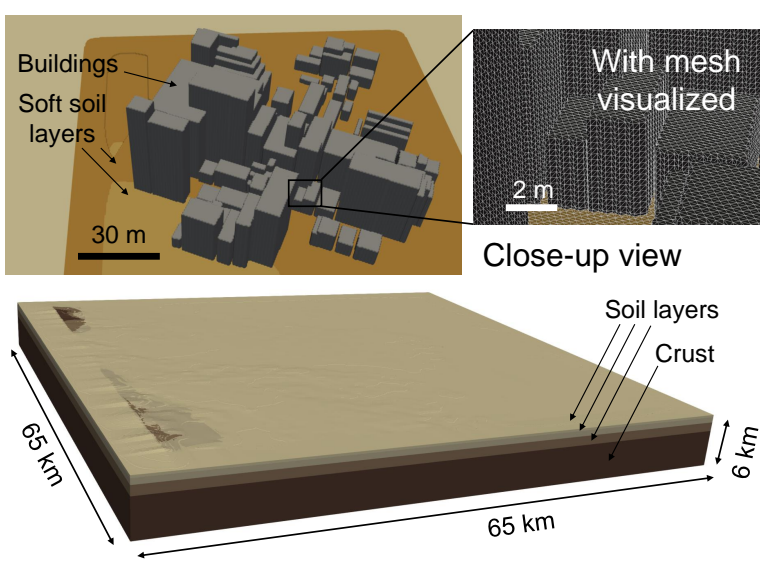

Figure 4: Performance measurement setting, created based on digital data of the actual crust, ground, and structures.

\subsection{Performance measurement environment}

We compare the large-scale computation performance of AEGIS on Fugaku. To demonstrate that the method is effective on a wide range of architecture, we also compare performance on a Xeon (Cascade Lake) CPU-based Oakbridge-CX system. At the kernel level, we also measure the performance on NVIDIA A100 GPU (PCI-E version), V100 GPU (NVLink version), and AMD EPYC (Rome) CPU.

Fugaku is a massively parallel supercomputer comprising 158,976 compute nodes, each with a single A64FX CPU [32] based on the Arm v8.2-A SVE instruction set architecture at RIKEN, Center for Computational Science $[33,34]$. Each of the 48 cores of the CPU has two sets of fused multiply-add (FMA) SIMD units with a width of 512 bits, leading to 3.072 FP64 TFLOPS hardware peak performance per node. The peak performance for FP32 is double the FP64 peak. Each node has 32 GB HBM2 memory with 1024 GB/s bandwidth. TofuD, a six-dimensional torus interconnection network, is used for communication between nodes [35]. Fujitsu Compiler/MPI environment tcsds-1.2.30a is used for measurement.

Oakbridge-CX (OBCX) [36] is a Xeon CPU server-based supercomputer system at Information Technology Center, The University of Tokyo, comprising 1368 compute nodes each with dual 28-core Xeon Platinum 8280 (Cascade Lake) CPUs. The CPU equips 512-bit SIMD units, leading to 4.84 TFLOPS hardware peak performance per node. Each node has 192 GB DDR4 memory with $281.6 \mathrm{~GB} / \mathrm{s}$ bandwidth. Compute nodes are connected with Intel Omni-Path Architecture with a full-bisection fat-tree topology. Intel Compiler 19.1.3.304 and Intel MPI version 2019 Update 9 is used for measurement.

Timers (MPI_WTIME()) were inserted in the code to measure elapsed time. Profilers fapppx and nvprof were used to measure FLOP counts on Fugaku and NVIDIA GPUs, respectively. The FLOP count on Xeon and AMD CPUs was approximated to be the same as the Arm program on Fugaku. 
Table 2: Configurations of the models used to measure performance (model set A: weak scaling, model set B: strong scaling).

\begin{tabular}{lrrrrrr}
\hline Model & $\begin{array}{r}\text { \# of compute } \\
\text { nodes }\end{array}$ & $\begin{array}{r}\text { \# of MPI } \\
\text { processes }\end{array}$ & Total DOF & $\begin{array}{r}\text { Mean DOF per } \\
\text { MPI domain }\end{array}$ & $\begin{array}{r}\text { Total \# of tetra } \\
\text { elements }\end{array}$ & $\begin{array}{r}\text { Total \# of tetra } \\
\text { elements in } C G_{p r e}^{\text {hmr }}\end{array}$ \\
\hline A-1 (Fugaku) & 578 & 2,312 & $9,391,390,881$ & $4,062,020$ & $2,337,732,136$ & $203,572,688$ \\
A-1 (OBCX) & 256 & 2,048 & $9,391,390,881$ & $4,585,640$ & $2,337,732,136$ & $203,572,688$ \\
A-2 & 1,156 & 4,624 & $18,780,893,655$ & $4,061,611$ & $4,675,464,272$ & $407,145,376$ \\
A-3 & 2,312 & 9,248 & $37,558,032,687$ & $4,061,205$ & $9,350,928,544$ & $814,290,752$ \\
A-4 & 4,624 & 18,496 & $75,112,399,539$ & $4,061,007$ & $18,701,857,088$ & $1,628,581,504$ \\
A-5 & 9,248 & 36,992 & $150,217,229,451$ & $4,060,803$ & $37,403,714,176$ & $3,257,163,008$ \\
A-6 & 18,496 & 73,984 & $300,427,127,835$ & $4,060,704$ & $74,807,428,352$ & $6,514,326,016$ \\
A-7 & 36,992 & 147,968 & $600,839,117,019$ & $4,060,601$ & $149,614,856,704$ & $13,028,652,032$ \\
A-8 & 73,984 & 295,936 & $1,201,663,095,387$ & $4,060,550$ & $299,229,713,408$ & $26,057,304,064$ \\
\hline B-1 & 289 & 1,156 & $9,391,390,881$ & $8,124,040$ & $2,337,732,136$ & $203,572,688$ \\
B-2 & 578 & 2,312 & $9,391,390,881$ & $4,062,020$ & $2,337,732,136$ & $203,572,688$ \\
B-3 & 1,156 & 4,624 & $9,391,390,881$ & $2,031,010$ & $2,337,732,136$ & $203,572,688$ \\
B-4 & 2,312 & 9,248 & $9,391,390,881$ & $1,015,505$ & $2,337,732,136$ & $203,572,688$ \\
\hline
\end{tabular}

\subsection{Performance on HPC architectures}

First, we measure the performance of the EBE kernel, which is highly architecture-dependent, on typical CPU and GPU architectures. Fig. $3 \mathrm{f}$ shows the performance of the EBE kernel on the A64FX CPU on Fugaku, Xeon CPU, EPYC CPU, A100 GPU, and V100 GPU. Here, the measurement is performed using the data of process numbers $0-3$ of Model A-1. Compared with the as-is EBE kernel developed in an SC14 Gordon Bell Prize finalist paper for the K computer, which had a narrow SIMD width of 2 and a core count of 8, the developed EBE kernel considering cache efficiency and wide SIMD showed a remarkable increase in execution performance, with a double-precision peak ratio of $12.3 \%$ on the A64FX on Fugaku and $21-22 \%$ on Xeon and EPYC CPUs. The double-precision peak ratio of the GPU version of the kernel, which uses a different approach to calculate EBE than the CPU version, was $22 \%$ and $19 \%$ on the A100 GPU and V100 GPU, respectively. Hence, high performance is achieved in a wide range of architectures by adopting kernel algorithms designed for each computer architecture. The performance on A64FX may not appear to be appealing at first glance, but this is because out-of-order resources are limited to improve power efficiency, and the memory bandwidth is designed to be sufficient; as described below, the full application, which includes memory bandwidth bound kernels, achieved significant performance.

Second, we measure the overall solver performance of $A E G I S$ and the state-of-the-art solver, $P C G E^{K}$, on Fugaku and Xeon-based OBCX (Fig. 5). Here, we measured performance on Model A-1 using 578 nodes of Fugaku (FP64 peak 1.77 PFLOPS, memory bandwidth $591 \mathrm{~TB} / \mathrm{s}$ ) and 256 nodes of OBCX (1.24 PFLOPS, $72.1 \mathrm{~TB} / \mathrm{s})$. As described below, the number of operations (FLOP) has been reduced to $1 / 8.16$ of the $P C G E^{K}$. Additionally, using the EBE kernel suitable for each architecture, high operation efficiency (8.14\% of FP64 peak for Fugaku and $7.12 \%$ for OBCX) has been obtained. As a result, 26.0- and 18.9-fold speedup were obtained from PCGE ${ }^{K}$ on Fugaku and OBCX, respectively. In the following, we analyze the details of the performance improvement factors. The total number of iterations for the $P C G E^{K}$ solver in solving for the 401-600 time steps was 62345 , whereas the total number of iterations for AEGIS was 43000, including $C G_{p r e} 4080, C G_{p r e}^{h m r} 8160$, and $C G_{p r e}^{l m r} 30760$. The accuracy of the initial solution estimation by Adams-Bashforth

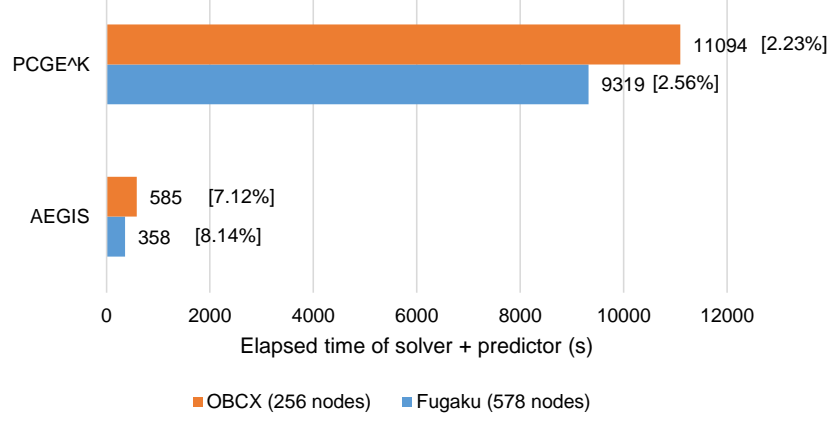

Figure 5: Comparison of solver + predictor execution times at Fugaku and OBCX for Model A-1. The FP64 peak ratio is shown in square brackets.

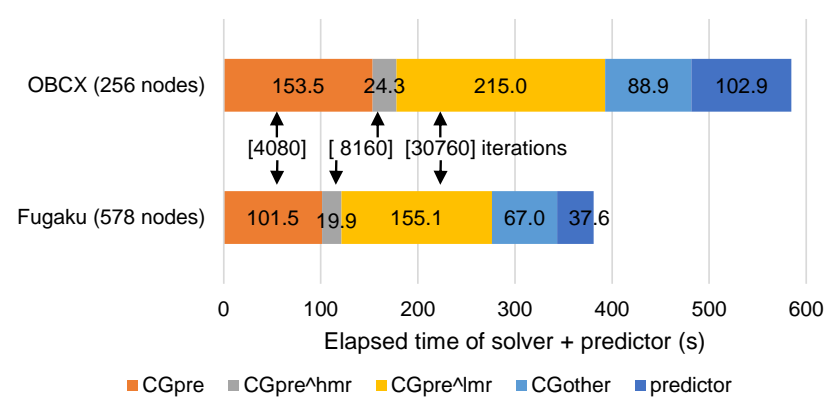

Figure 6: Breakdown of elapsed time of $A E G I S$ on Model A1. Note that the predictor is data access bandwidth bound, and thus relatively high performance is achieved on Fugaku with higher memory bandwidth.

used in $P C G E^{K}$ (relative error of about $10^{-3}$ ) is greatly improved by the predictor of $A E G I S$ (relative error of about $10^{-5}$ ), and the lower frequency mode is efficiently solved by the coarse grid $\left(C G_{p r e}^{\operatorname{lm} r}\right)$ in the multigrid, which are thought to result in the reduction of the number of iterations. Considering that the ratio of DOF of the 
CG iterations of $P C G E^{K}$ and each iteration of $C G_{p r e}, C G_{p r e}^{h r r}$, and $C G_{p r e}^{l m r}$ in AEGIS is 1:1, 1:0.087, and 1:0.125, respectively, the number of floating point operations decreases more than the ratio of the number of iterations, and the number of operations becomes $1 / 8.16$. The breakdown of the execution time shown in Fig. 6 illustrates that the execution time per iteration for $C G_{p r e}^{\operatorname{lm} r}$ and $C G_{p r e}^{h m r}$ has been reduced compared to $C G_{p r e}$, as expected. Additionally, the predictor takes only approximately $1 / 10$ of the total execution time, indicating that the effects of reducing the number of iterations, reducing the number of operations per iteration, and improving the efficiency of operations have resulted in the speedup on both systems.

\subsection{Performance on large-scale problems}

Next, we measure the performance of AEGIS on a large-scale problem in Fugaku, which is presently the largest computing environment (Fig. 7). First, AEGIS shows good time to solution and scalability compared to other methods. Specifically, compared with $P C G E^{K}$, which is considered the state-of-the-art, the speedup is $9318 / 366=$ 25.45 times faster even in Model A-3. Since $P C G E^{K}$ requires long duration runs and extremely large computational resources to be measured for large-scale problems, we developed $P C G E^{F u g a k u}$, which incorporates the EBE kernel developed in this study to accelerate the state-of-the-art solver, and solved larger-scale problems. However, its speedup was only 9318/1718 $=5.42$ times in Model A-3, which is considerably slower than that of $A E G I S$, and the speedup of $A E G I S$ is superior. Conversely, we attempted to solve a large-scale problem using GAMERA ${ }^{F u g a k u}$, which was developed by incorporating the EBE kernel developed in this study into GAMERA ${ }^{K}$, which was initially expected to be an state-of-the-art solver. However, the number of iterations increased with the problem size and the number of computer nodes, and the solver failed to converge on more than 36,992 nodes. This was a problem of taking care of lowprecision computation when solving large-scale problems; it was found that the increase in the number of iterations in large-scale problems could be suppressed by calculating the preconditioning of GAMERA in double precision. In other words, the accuracy of preconditioning using low-precision arithmetic was insufficient for complex problems with widely varying characteristics targeted in this study. Note that GAMERA ${ }^{K}$ and GAMERA ${ }^{\text {Fugaku }}$ are inferior to $A E G I S$ in that they are slow even for small-scale problems and cannot solve large-scale problems. Conversely, as in PCGE $E^{F u g a k u}$, which calculates the entire process in double precision, the number of iterations is maintained flat up to high parallelism in AEGIS by the suppression of errors due to low-precision operations, and high weak scaling performance is achieved even in large-scale models. Since the total number of iterations and the associated number of MPI_Allreduce and communication between neighboring nodes are reduced in $A E G I S$, the speedup of AEGIS increased from 4.73-fold of PCGE ${ }^{F u g a k u}$ on the smallest Model A-1 to 5.04-fold of PCGE ${ }^{\text {Fugaku }}$ in the largest comparable Model A-7. The weak scaling from Model A-1 to Model A-8 was $93.7 \%$, and the peak performance for Model A-8 was 17.07 PFLOPS (7.51\% of the hardware peak). Additionally, as shown in Fig. 8, measurements on a large number of nodes show that AEGIS achieves high strong scaling

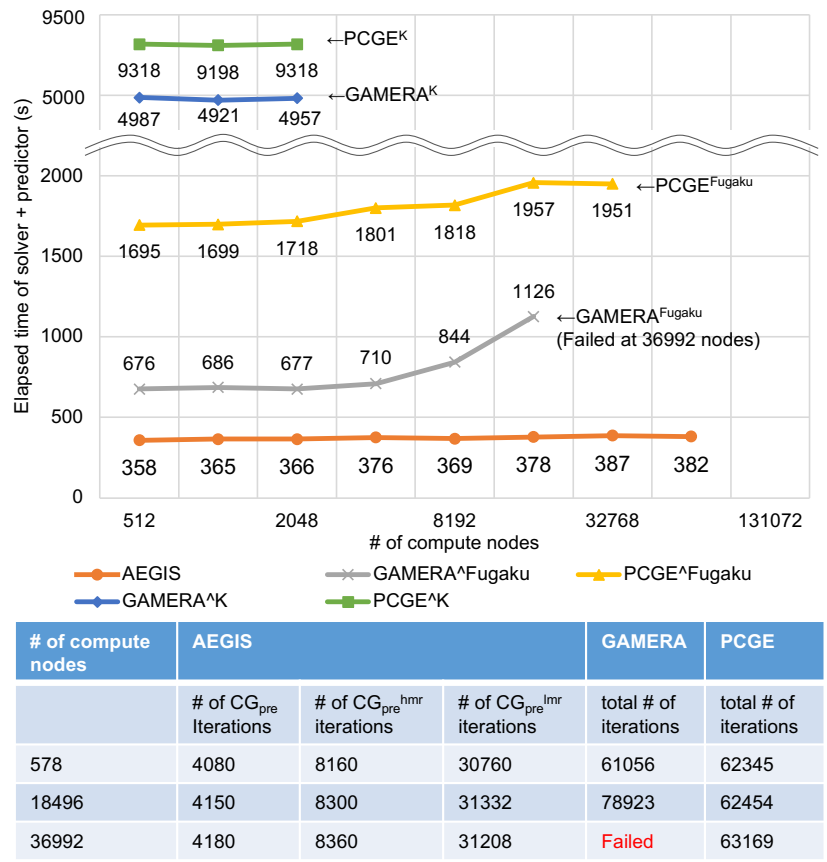

Figure 7: Size up scalability for model set A.

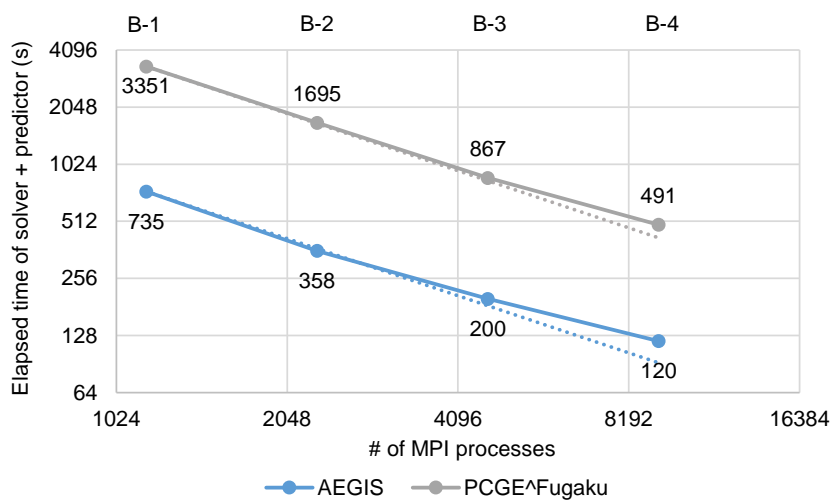

Figure 8: Speedup scalability for model set B. Note that only problem sizes up to $B-3$ is used for actual use purposes and thus B-4 is only for a reference aimed for shortest time-tosolution.

performance and a high speedup ratio compared to PCGE even when the problem scale per compute node is small.

\subsection{Performance on actual applications}

We confirm the performance of AEGIS using a realistic application example of 324 billion DOF shown in Section 1, which is difficult to achieve high performance. As the operation of the entire system of Fugaku is planned to be limited to a few days per year, being able to solve the application problem under the normal operation of Fugaku 
(up to 55,296 nodes for $24 \mathrm{~h}$ ) is crucial for analyzing various earthquake scenarios; thus, we first check the performance of AEGIS on 52,992 nodes of Fugaku. We would like to use $P C G E^{K}$ for comparison, but it is too slow even on Fugaku, so we use PCGE $E^{F u g a k u}$, which is a faster version of $P C G E^{K}$ with the EBE developed in this study. On average of the time steps 1500-5700 available for comparison, $A E G I S$ was $4.4-$ fold faster than $P C G E^{F u g a k u}$; if comparison with state-of-the-art solver $P C G E^{K}$ was possible, $A E G I S$ would have been 23.87-fold faster (performance ratio of $9318 \mathrm{~s}\left(P C G E^{K}\right) / 1718$ $\mathrm{s}\left(P C G E^{F u g a k u}\right)=5.42$-fold obtained in the performance measurement problem is used). Additionally, AEGIS could analyze up to 9550 steps in $4 \mathrm{~h}$ and $36 \mathrm{~min}$, which is extremely fast at $1.5 \mathrm{~s}$ per time step. This means that we can now compute complex Terra problems with at least 1 Tera DOF in normal operation, which is far beyond what was able in the previously possible. By contrast, state-of-the-art solver $P C G E^{K}$ is estimated to take $4.6 \times 23.87=$ $109.8 \mathrm{~h}$, indicating that analyzing such an application with state-ofthe-art solver $P C G E^{K}$ is impractical, even on Fugaku. The speedup ratio of AEGIS/PCGE Fugaku in this application is almost the same as that in the performance measurement problem (4.7-5.0 times), demonstrating that our method is fast and robust even for practical application problems with more complex source processes than the performance measurement problem. This is because the error of the initial solution by the predictor of AEGIS is one to two orders of magnitude lower than that of the Adams-Bashforth method at almost all time steps and because of the effect of multigrid preconditioning with low-precision operations. Finally, the computation of the application example using 152,352 computer nodes (609,408 MPI processes $\times 12$ OpenMP threads $=7,312,896$ parallel computation) aiming for the fastest time to solution, disregarding performance efficiency and scalability, resulted in a further 1.94-fold speedup from the above-mentioned 52,992 node case with an effective performance of $4.66 \%$ (21.8 PFLOPS) although the DOF per computer node was small $(4.66 \%$ is significantly better than that of ordinary finite-element solvers on low ordered unstructured elements, and for the case with larger problem size per node, the efficiency is expected to improve as in Model A- 8 of the performance measurement model on 73,984 computer nodes).

\section{CONCLUDING REMARKS}

We have developed an implicit solver AEGIS that scales to $152 \mathrm{~K}$ computer nodes $(609,408$ MPI processes $\times 12$ OpenMP threads $=$ $7,312,896$ parallel computation) and realized super-large dynamic nonlinear finite element analysis using 3D unstructured low-order elements. The proposed method, which achieved a 25.45 -fold speedup from the state-of-the-art solver on Fugaku, provided a complete solution to a problem that the SC15 Gordon Bell Prize Finalist solver on K computer could not touch. When comparing the state-of-theart solver@K computer and the proposed method@Fugaku, a huge leap is enabled by the 42 -fold hardware performance improvement $\times$ the 25.4-fold solver method improvement $=1070$-fold improvement. Fully coupled analysis of ultra-high-fidelity fault-structure systems is one of the dreams of earthquake engineering, and this analysis demonstrates that it is now feasible and that Terra can be solved with Tera DOF; we can expect it to become one of the milestones in future earthquake engineering simulations. Additionally, this method can be applied to time evolution problems with higherorder modes and poor convergence; thus, for many problems, a speedup is expected. In particular, the initial solution estimator by massively parallel data learning using data generated during analysis is highly versatile and can be easily ported to other solvers. Moreover, demonstrating the method for accelerating computationally rich kernels, including data recurrence, which frequently occurs in other applications, as generalized on each architecture shows the versatility of this solver. It is expected to contribute to the speeding up of similar kernels for other applications.

A robust and high-speed system for conducting detailed analysis in a wider area through trial and error, as well as timely analysis of the obtained results by the strengthening of the file system and visualization flow to make a decision, is desirable for future system development from the viewpoint of the application development side. For example, excessive time is spent on MPI initialization and file I/O in the current analysis using hundreds of thousands of nodes or MPI processes; hence, in future system design, a mechanism that can manage I/O and visualization hierarchically with fat nodes that can perform high-speed analyses while suppressing the number of nodes or processes is desirable. Cooperation between system and application developers will become more important as systems become more sophisticated and complex, and co-designing systems and applications should be continued.

\section{ACKNOWLEDGMENTS}

Results were obtained using Fugaku at Center for Computational Science, RIKEN, and Oakbridge-CX at Information Technology Center, The University of Tokyo. This work was supported by MEXT as "Program for Promoting Researches on the Supercomputer Fugaku" (Large-scale numerical simulation of earthquake generation, wave propagation and soil amplification: hp200126, hp210171) and as "Post K computer project" (Priority Issue 3 - Development of integrated simulation systems for hazards and disasters induced by earthquakes and tsunamis: hp190177) and JSPS KAKENHI Grant Numbers JP18H05239, JP18H03795, JP17K14719. We thank Youji Matsumoto of Cybernet Systems Co., Ltd. for support in visualizing the application example.

\section{REFERENCES}

[1] T.H. Jordan, "Societal Impact of Earthquake Simulations at Extreme Scale," SC '15 Invited Talk Spotlight.

[2] L. Carrington, D. Komatitsch, M. Laurenzano, M. Tikir, D. Michea, N.L. Goff, A. Snavely, and J. Tromp, "High-frequency simulations of global seismic wave propagation using SPECFEM3DGLOBE," Proceedings of SC '08, pp. 1-11, 2008.

[3] M. Rietmann, P. Messmer, T. Nissen-Meyer, D. Peter, P. Basini, D. Komatitsch, O. Schenk, J. Tromp, L. Boschi, and D. Giardini, "Forward and adjoint simulations of seismic wave propagation on emerging largescale GPU architectures," Proceedings of SC '12, pp. 1-11, 2012.

[4] Y. Cui, E. Poyraz, K. B. Olsen, J. Zhou, K. Withers, S. Callaghan, J. Larkin, C. Guest, D. Choi, A. Chourasia, Z. Shi, S. M. Day, P. J. Maechling, and T. H. Jordan, "Physics-based seismic hazard analysis on petascale heterogeneous supercomputers," Proceedings of SC '13, pp. 1-12, 2013.

[5] A. Heinecke, A. Breuer, S. Rettenberger, M. Bader, A-A. Gabriel, C. Pelties, A. Bode, W. Barth, X-K. Liao, K. Vaidyanathan, M. Smelyanskiy, and P. Dubey, "Petascale High Order Dynamic Rupture Earthquake Simulations on Heterogeneous Supercomputers," Proceedings of SC '14, pp. 3-14, 2014.

[6] D. Roten, Y. Cui, K.B. Olsen, S.M. Day, K. Withers, W.H. Savran, P. Wang, and D. $\mathrm{Mu}$, "High-frequency nonlinear earthquake simulations on petascale heterogeneous supercomputers," Proceedings of SC '16, pp. 957-968, 2016.

[7] H. Fu, C. He, B. Chen, Z. Yin, Z. Zhang, W. Zhang, T. Zhang, W. Xue, W. Liu, W. Yin, G. Yang, and X. Chen, "15-Pflops Nonlinear Earthquake Simulation on Sunway 
TaihuLight: Enabling Depiction of Realistic $10 \mathrm{~Hz}$ Scenarios," Proceedings of SC '17, article No. 2, 2017

[8] C. Uphoff, S. Rettenberger, M. Bader, E. H. Madden, T. Ulrich, S. Wollherr, and A.-A. Gabriel, "Extreme scale multi-physics simulations of the tsunamigenic 2004 sumatra megathrust earthquake," Proceedings of SC '17, pp. 1-16, 2017.

[9] T. Ichimura, K. Fujita, S. Tanaka, M. Hori, M. Lalith, Y. Shizawa, and H. Kobayashi "Physics-based urban earthquake simulation enhanced by 10.7 BlnDOF x $30 \mathrm{~K}$ time-step unstructured FE non-linear seismic wave simulation," Proceedings of SC '14, pp. 15-26, 2014.

[10] T. Ichimura, K. Fujita, P. E. B. Quinay, L. Maddegedara, M. Hori, S. Tanaka, Y Shizawa, H. Kobayashi, and K. Minami, "Implicit Nonlinear Wave Simulation with 1.08T DOF and 0.270T Unstructured Finite Elements to Enhance Comprehensive Earthquake Simulation," Proceedings of SC '15, pp. 1-12, 2015.

[11] T. Ichimura, K. Fujita, T. Yamaguchi, A. Naruse, J. C. Wells, T. C. Schulthess, T. P. Straatsma, C. J. Zimmer, M. Martinasso, K. Nakajima, M. Hori, and L. Maddegedara, "A fast scalable implicit solver for nonlinear time-evolution earthquake city problem on low-ordered unstructured finite elements with artificial intelligence and transprecision computing," Proceedings of SC '18, article No. 49, 2018.

[12] N. M. Newmark, "A method of computation for structural dynamics," Journal of the Engineering Mechanics Division, 85 (EM3), pp. 67-94, 1959.

[13] Y.D. Isbiliroglu, R. Taborda, and J. Bielak, "Coupled soil-structure interaction effects of building clusters during earthquakes," Earthq. Spectra, 31, pp. 463-500, 2013.

[14] W. Zhang, D. Restrepo, J. G.F. Crempien, B. Erkmen, R. Taborda, A. Kurtulus, and E. Taciroglu, "A computational workflow for rupture-to-structural-response simulation and its application to Istanbul," Earthquake Engineering Structural Dynamics, 50, pp. 177-196, 2021.

[15] T. Ichimura, M. Hori, P. E. B. Quinay, L. Maddegedara, T. Suzuki, and S. Noguchi, "Comprehensive numerical analysis of fault-structure systems - Computation of the large-scale seismic structural response to a given earthquake scenario -," Earthquake Engineering \& Structural Dynamics, 41, pp 795-811, 2012.

[16] The American Society of Mechanical Engineers, Guide for verification and validation in computational solid mechanics, 2006.

[17] T. Ichimura, M. Hori, and J. Bielak, "A Hybrid Multiresolution Meshing Technique for Finite Element Three-Dimensional Earthquake Ground Motion Modeling in Basins Including Topography," Geophysical Journal International, 177, pp 1221$1232,2009$.

[18] K. Fujita, K. Katsushima, T. Ichimura, M. Horikoshi, K. Nakajima, M. Hori, and L. Maddegedara, "Wave propagation simulation of complex multi-material problems with fast low-order unstructured finite-element meshing and analysis," Proceedings of the International Conference on High Performance Computing in Asia-Pacific Region (HPC Asia 2018), pp. 24-35, 2018.

[19] GEBCO Bathymetric Compilation Group 2020, The GEBCO_2020 Grid - a continuous terrain model of the global oceans and land, British Oceanographic Data Centre, National Oceanography Centre, 2020.

[20] K. Fujita, K. Koyama, K. Minami, H. Inoue, S. Nishizawa, M. Tsuji, T. Nishiki, T. Ichimura, M. Hori, and L. Maddegedara, "High-fidelity nonlinear low-order unstructured implicit finite-element seismic simulation of important structures by accelerated element-by-element method," Journal of Computational Science, 49, article No. 101277, 2021.

[21] T. Ichimura, K. Fujita, K. Koyama, R. Kusakabe, K. Minami, H. Inoue, S. Nishizawa M. Tsuji, T. Nishiki, M. Hori, L. Maddegedara, and N. Ueda, "Fast Scalable Implicit Solver with Convergence of Physics-Based Simulation and Data-Driven Learning," Research Poster for SC '20, 2020.

[22] J.M. Winget and T.J. Hughes, "Solution algorithms for nonlinear transient heat conduction analysis employing element-by-element iterative strategies," Computer Methods in Applied Mechanics and Engineering, 52, pp. 711-815, 1985.

[23] "EBE kernel for A64FX CPUs [Online]." To be available.

[24] J. N. Kutz, S. L. Brunton, B. W. Brunton, and J. L. Proctor, Dynamic Mode Decomposition: Data-Driven Modeling of Complex Systems, Society for Industrial \& Applied Mathematic, 2016

[25] G. H. Golub and Q. Ye, "Inexact preconditioned conjugate gradient method with inner-outer iteration," SIAM Journal on Scientific Computing, 21, pp. 1305-1320, 1999.

[26] M. Kubale, Graph Colorings, American Mathematical Society, 2004.

[27] C. Cecka, A. Lew, E. Darve, "Assembly of finite element methods on graphics processors," Int. J. Numer. Meth. Eng., 85, pp. 640-669, 2011.

[28] G. Karypis and V. Kumar, "A fast and high quality multilevel scheme for partitioning irregular graphs," SIAM Journal on scientific Computing, 20, pp. 359-392, 1998.

[29] "Strong ground motion of The Southern Hyogo prefecture earthquake in 1995 observed at Kobe JMA observatory, Japan Meteorological Agency, [Online]." https://www.data.jma.go.jp/svd/eqev/data/kyoshin/jishin/

[30] I.M. Idriss, R. Dobry, and R.D. Sing, "Nonlinear behavior of soft clays during cyclic loading," J. Geotech. Eng. Div, 104, pp. 1427-1447, 1978.

[31] G. Masing, "Eigenspannungen und Verfestigung beim Messing," Proceedings of the 2nd International Congress of Applied Mechanics, pp. 332-335, 1926.
[32] T. Yoshida, "Fujitsu High Performance CPU for the Post-K Computer," IEEE Hot Chips: A Symposium on High Performance Chips, 2018.

[33] "Supercomputer Fugaku, RIKEN Center for Computational Science [Online]." https://www.r-ccs.riken.jp/en/fugaku/

[34] M. Sato, Y. Ishikawa, H. Tomita, Y. Kodama, T. Odajima, M. Tsuji, H. Yashiro, M. Aoki, N. Shida, I. Miyoshi, K. Hirai, A. Furuya, A. Asato, K. Morita, and T. Shimizu, "Co-design for A64FX manycore processor and "Fugaku"," Proceedings of SC '20, pp. 1-15, 2020.

[35] Y. Ajima, T. Kawashima, T. Okamoto, N. Shida, K. Hirai, T. Shimizu, S. Hiramoto, Y. Ikeda, T. Yoshikawa, K. Uchida, and T. Inoue, "The Tofu Interconnect D," 2018 IEEE International Conference on Cluster Computing (CLUSTER), pp. 646-654, 2018.

[36] "Oakbridge-CX Supercomputer System, Information Technology Center, The University of Tokyo [Online]." https://www.cc.utokyo.ac.jp/en/supercomputer/obcx/service/ 